\title{
An evaluation of Drosophila as a model system for studying tauopathies such as Alzheimer's disease
}

Sivanantharajah, Lovesha; Mudher, Amritpal; Shepherd, David

\section{Journal of Neuroscience Methods}

DOI:

10.1016/j.jneumeth.2019.01.001

Published: 01/05/2019

Peer reviewed version

Cyswllt i'r cyhoeddiad / Link to publication

Dyfyniad o'r fersiwn a gyhoeddwyd / Citation for published version (APA):

Sivanantharajah, L., Mudher, A., \& Shepherd, D. (2019). An evaluation of Drosophila as a model system for studying tauopathies such as Alzheimer's disease. Journal of Neuroscience Methods, 319(May), 77-88. https://doi.org/10.1016/j.jneumeth.2019.01.001

\footnotetext{
Hawliau Cyffredinol / General rights

Copyright and moral rights for the publications made accessible in the public portal are retained by the authors and/or other copyright owners and it is a condition of accessing publications that users recognise and abide by the legal requirements associated with these rights.

- Users may download and print one copy of any publication from the public portal for the purpose of private study or research.

- You may not further distribute the material or use it for any profit-making activity or commercial gain

- You may freely distribute the URL identifying the publication in the public portal ?
}

Take down policy

If you believe that this document breaches copyright please contact us providing details, and we will remove access to the work immediately and investigate your claim. 
An evaluation of Drosophila as a model system for studying tauopathies such as Alzheimer's disease

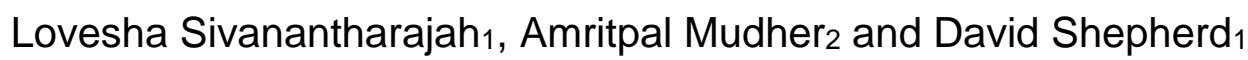

${ }^{1}$ School of Natural Sciences, Bangor University, Bangor, Gwynedd, UK

2 Faculty of Natural and Environmental Sciences, University of Southampton, Southampton, UK

Corresponding author: Lovesha Sivanantharajah (I.sivanantharajah@bangor.ac.uk) 


\begin{abstract}
Work spanning almost two decades using the fruit fly, Drosophila melanogaster, to study tau-mediated neurodegeneration has provided valuable and novel insights into the causes and mechanisms of tau-mediated toxicity and dysfunction in tauopathies such as Alzheimer's Disease (AD). The fly has proven to be an excellent model for human diseases because of its cost efficiency, and the availability of powerful genetic tools for use in a comparatively less-complicated, but evolutionarily conserved, in vivo system. In this review, we provide a critical evaluation of the insights provided by fly models, highlighting both the advantages and limitations of the system. The fly has contributed to a greater understanding of the causes of tau abnormalities, the role of these abnormalities in mediating toxicity and/or dysfunction, and the nature of causative species mediating tautoxicity. However, it is not possible to perfectly model all aspects of human degenerative diseases. What sets the fly apart from other animal models is its genetic tractability, which makes it highly amenable to overcoming experimental limitations. The explosion of genetic technology since the first fly disease models were established has translated into fly lines that allow for greater temporal control in restricting tau expression to single neuron types, and lines that can label and monitor the function of subcellular structures and components; thus, fly models offer an unprecedented view of the neurodegenerative process. Emerging genetic technology means that the fly provides an ever-evolving experimental platform for studying disease.
\end{abstract}

Keywords: (6) animal models, Drosophila, neurodegenerative diseases, tauopathy, Alzheimer's disease 


\section{Introduction}

\subsection{Is the fly a good model to study neurodegeneration?}

The first Drosophila models analysing the effects of human tau (htau) on the fly nervous system stemmed from work by Williams et al. (2000) and Wittman et al. (2001). Williams et al. (2000) showed that expression of a variety of tau fusion proteins, including htau or bovine tau, in adult sensory neurons produced morphological phenotypes such as axon blebbing, axon loss, axon defasciculation, and reduced arborization that were consistent with a degenerative process. Wittman et al. (2001) subsequently showed that expression of wild type and mutant tau proteins had a significant effect on longevity with an obvious neurodegenerative outcome that was more pronounced with disease-causing mutant tau proteins. These two studies were the earliest to document the neurodegenerative effects of tau overexpression in fly neurons, which appeared to recapitulate disease pathology phenotypes observed in tauopathies like Alzheimer's disease (AD). Coupled with the demonstration that neurodegeneration associated with other neurodegenerative diseases, such as Parkinson's disease (Feany and Bender 2000) and Huntington's disease (Jackson et al. 1998), could be simulated in flies, Drosophila was established as a credible animal model for studying the cellular and molecular mechanisms of human diseases. Building on these studies the use of Drosophila as a disease model has expanded rapidly and played an important role in furthering our understanding of the role of tau in pathogenesis and progression of $A D$, and other tauopathies.

\subsubsection{Deep evolutionary conservation}

At a superficial level the fly may not seem an obvious model for studying human neurogenerative diseases, but closer examination of shared biology reveals more similarities than differences with humans. There is a large body of evidence demonstrating a deep evolutionary conservation in animal nervous systems, and the Drosophila and human nervous systems show a remarkable degree of molecular and cellular conservation. Fundamental developmental processes and principles used by both flies and humans to build a complex nervous system descended from a shared common ancestor that lived roughly 570 million years ago. This ancestor was already equipped with a fully functioning nervous system with all the elements that we would recognise today (Denes et al. 2007), consequently, flies and humans share similar neuron morphology, physiology, transmitter systems, cell biology and neurochemistry.

At the cellular level, all the cellular machinery of mammalian neurons is conserved in flies with homologous proteins and processes underlying the structure of the cytoskeleton, axon transport processes, synaptic transmission, neuron excitability, and signal transduction. These homologies are not just restricted to cellular components, at the molecular level many fundamental genetic programs that lay the body plan and dictate how the nervous system is built are conserved. For example, differentiation and determination of neuronal identity is controlled by the same conserved family of transcription factors, the Homeotic selector (Hox) genes, that pattern neuronal identity (i.e. establish different neuron subtypes) along the anterior-posterior body axis in both flies and humans (reviewed by Philippidou and Dasen 2013).

Despite the cellular and molecular homologies, mammalian and insect nervous systems are often seen as being fundamentally different in the way in which they are organised and assembled during development; however, even here there is increasing evidence that aspects of brain organisation and development are also conserved. This ranges from the mechanisms that specify the neuroaxis and the development of the 
different brain regions (Arendt et al. 2008, 2016, Denes et al. 2007) to the way in which neurons are generated. In both flies and mammals, neurons are organised into functionally specialized groups, with similar functional roles and properties, sharing the same developmental origins. The stem cells generating the pools of functionally similar interneurons in the vertebrate spinal cord appear to be conserved with the progenitor cells and transcription factors that define different interneuron classes conserved from lower vertebrates to mammals (Lupo et al. 2006) and insects (Lacin and Truman 2016, Harris et al. 2015). Perhaps more controversial, there is evidence that the structure of the nervous system may also be conserved with growing evidence of homology between structures in the fly and mammalian brains with conserved roles, one example being brain regions associated with learning and memory (Wolff and Strausfeld 2016).

In summary, this level of evolutionary conservation is important because it allows more accurate modelling of pathways that can contribute to disease pathogenesis and progression. Though cellular environments between flies and humans may be not be identical, they are certainly similar enough to allow modelling of human disease in a comparatively simple animal.

\subsubsection{Comparative simplicity}

The fundamental feature of the fly that gives it such utility as a model for human diseases such as $A D$, is the conservation of disease related genes. It has long been known that $75 \%$ of the human genes known to be associated with neurological conditions have orthologues in flies (Bier 2005). Given the relative simplicity of the fly genome compared to the human genome, which has a high level of functional genetic redundancy, the function of novel disease related genes can be more easily dissected and understood in the fly. Where in humans and rodents there are often multiple isoforms of the proteins associated with disease, in flies there are often fewer isoforms. How true this is for tau is not entirely clear, because though numerous tau-like polypeptides are predicted in flybase (http://flybase.org/reports/FBgn0266579) there is evidence verifying just one primary isoform and suggesting another larger isoform present at much lower levels (Heidary and Fortini 2001, Burnouf et al. 2016). Nonetheless, this is still fewer than those found in vertebrates: three isoforms identified in rodents (Mavilia et al. 1993) and six isoforms expressed in the central nervous system (CNS) of humans (Ksiezak-Reding et al.1988). Less genetic redundancy in flies means that one is less likely to see compensatory effects of multiple isoforms when studying the role of any one isoform in disease using fly models. Comparative simplicity also extends to the nervous system, which in adult flies is composed of roughly 100,000 neurons. This has already contributed to detailed connectivity maps of fly neurons with single-cell resolution (Takemura et al. 2017a, Takemura et al. 2017b). Soon it will be possible to examine the effects of pathogenic proteins at the level of an individual neuron and its connections or even on a larger scale-an entire connectome.

\subsubsection{Experimental tractability}

An advantage of the fly as an experimental system is that it is not limited by some of the considerations that affect mammalian systems, such as costs, care and time. Cell culture models can offer a cost-effective alternative to rodent models, but they are limited for probing behaviour and systems-level responses. Advancement with organoids will undoubtedly present better solutions for recreating a multicellular environment to study disease, but organoids are still in vitro models and have some of the limitations of standard cell culture techniques. In contrast to this, the fly is an inexpensive, time-efficient, genetically tractable model that permits detailed, in vivo dissection of the mechanisms 
underpinning pathogenesis and progression of disease, whilst permitting impact on behavioural outputs. Furthermore, the genetic tool kit available in the fly gives it a value that is hard to parallel in any other multicellular system with a complex central brain. In flies it is possible to model many facets of tau pathology in vivo, such as cell death and tau hyperphosphorylation. It is also possible to study how these pathologies influence complex behaviours affected in human disease, such as memory dysfunction, circadian behaviour, and locomotor function using standardized and well-characterised behavioural assays (Ugur et al. 2016). Nonetheless, not all pathological aspects of disease can be emulated in flies, though sometimes this may itself prove informative. For example, some disease elements like the formation of insoluble, tau aggregates and tangles are not observed in flies (except in particular experimental scenarios discussed later). While this might seem to be a limitation of the fly it may in fact be revealing, suggesting that tau aggregation per se is not necessary for tau-mediated toxicity and dysfunction, since these fly models display neurodegeneration even in the absence of tau aggregation. Rather, these models imply that aggregation could be a late stage or end stage pathology. Indeed, this is now reiterated by numerous rodent models as well (reviewed in Cowan and Mudher 2013).

A key consideration when assessing the value of the fly, or any other model, is to understand how each model is suited to addressing particular questions rather than how accurately it emulates human disease. For example, expressing tau in motor neurons of larval Drosophila creates a locomotor phenotype, which in itself may not be relevant to AD (even though it is a feature of many other tauopathies). However, by expressing tau specifically in motor neurons it is possible to ask how abnormal tau disrupts neuronal function in a neuronal circuit that is ideal for probing neuronal structure and functional defects (e.g. using axonal transport and synaptic integrity assays)(Chee et al. 2005), which culminate in a quantifiable behavioural output (e.g. locomotor assays) (Mudher et al. 2004). This information may be extrapolated to shed light on how neuronal function may be altered by pathological tau in other circuits affected in tauopathies.

As with all other models of disease, there are some elements of disease that simply cannot be modelled in a fly due to different life histories and physiological systems. Foremost is time; flies live for just 10 weeks and is not possible to study all the impacts of longevity on the disease process. Whilst some aspects of longevity such as aging and the progressive nature of tau phenotypes can be studied in flies, for other aspects of disease that seem to require long periods for maturation (e.g. tau aggregation into large filaments and tangles) the life span of the fly may not be sufficient. Similarly, the more divergent elements of fly systems make it more difficult to analyse the broader physiological aspects of the disease that involve for example adaptive immunity, inflammatory processes or pharmacodynamics. For the latter, the mode of drug action may be studied, or a drug target may be identified; however, it is not possible to study drug delivery, metabolism, half-life or its removal. Evidently the use of flies is limited to those elements that can be meaningful in such a simple, short-lived system. Key in this regard is an appreciation that fly models of tauopathy allow one aspect of tau pathology to be simulated in a simple organism so that fundamental questions about how this pathology arises and how it impacts neuronal structure and function can be addressed. It is important to realise that the aim of working with the fly is not to create Alzheimer's disease in a model, but to emulate molecular elements of the disease in order to understand the mechanisms that contribute to the neuronal dysfunction and neurodegeneration that gives rise to the clinical symptoms in that complex human condition. 


\subsection{The genetic tools that allow modelling of disease in Drosophila}

All models of neurodegenerative disease in Drosophila rely on the GAL4-UAS binary expression system that allows for temporal and spatial control of gene expression (Brand and Perrimon 1993). The GAL4 system comprises two parts: the GAL4 "driver" line, in which an enhancer element drives expression of the yeast transcriptional activator, GAL4, in a reproducible temporal and spatial pattern, and the UAS "responder" line, in which the sequence of a gene of interest is inserted downstream of the GAL4 Upstream Activation Sequence (UAS). Crossing driver and responder lines to combine both elements in the same fly results in GAL4 binding to the UAS sequence and initiating expression of a gene of interest in a pattern dictated by the driver line. Traditionally these two elements have been randomly inserted into the fly genome using p-element mediated transposition (Brand and Perrimon 1993); however, site-directed methods using flippases and CRISPR-Cas9 based genome editing allow insertion of transgenes into specific genomic locations (Bischof et al. 2007, Jinek et al. 2012). These latter developments are useful for controlling expression level differences between constructs that may arise from random insertion methods, making comparative studies of different constructs more consistent.

\subsubsection{GAL4-responder lines to express tau and other effectors:}

The key to fly work is that "engineering" a fly to create a transgenic stock incorporating a novel UAS responder construct is relatively easy, inexpensive and often commercially outsourced, meaning that it is theoretically possible to target the expression of any disease related human protein in flies. However, a wealth of extant genetic tools is readily available in the fly community and stock centres such as the Bloomington Drosophila Stock Centre (BDSC, http://flystocks.bio.indiana.edu). For example, there are a number of fly models of tauopathy available that allow expression of different variants of htau in the fly's nervous system and offer well-established, quantifiably assays for detecting causal effects. This includes, but is not limited to, GAL4 lines for expressing Drosophila tau (dtau) (Mershin et al. 2004), bovine tau (Ito et al. 1997), any of the six wildtype isoforms of human tau (Williams et al. 2000, Wittmann et al. 2001, Chatterjee et al. 2009), mutations at tau phosphorylation sites specific for particular kinases (Chatterjee et al. 2009, Nishimura et al. 2004), mutations that mimic constitutively dephosphorylated or pseudophosphorylated states of tau (Khurana et al. 2006) and mutations associated with other tauopathies (e.g. $\mathrm{R} 406 \mathrm{~W}$ tau which is a mutation associated with frontotemporal lobe dementia (FTD) (Wittmann et al. 2001). These models have undoubtedly made enormous contributions to our understanding of tauopathies, but one could argue that like similar rodent models, there are two inherent traits that should be borne in mind when interpreting results: all these models invariably express the htau in flies already expressing endogenous dtau, and the htau gene may potentially be "over-expressed" because there is little information about how the htau expression compares to that of the endogenous dtau. How htau impacts endogenous dtau, and whether or not this contributes to the htau phenotype is not always clear, though some studies have examined this (Cowan et al. 2010a). Such issues affected rodent models of tauopathy for decades and have led to newer rodent models wherein the htau is expressed in transgenics in which the rodent tau has been removed. This approach is also being adopted by Drosophila biologists, who are beginning to create similar "humanised" fly models - in these newer fly models, dtau is deleted and the htau gene is "knocked-in" into the dtau locus (Gorsky et al. 2017). Expression of htau is then controlled by endogenous dtau regulatory elements. It is conceivable that these newer models may enable an even closer emulation of disease because they will achieve physiological htau expression without any confounding influence (if any) of endogenous dtau. 
Stock centres such as the BDSC also offer thousands of useful UAS responder lines that allow fluorescent labelling of entire cells, subcellular compartments (e.g. nucleus, cytoplasm, dendrites, synapses, etc.) and subcellular components (e.g. organelles such as mitochondria, labelling of endosomes, lysosomes etc.). These reagents are useful for assaying cell structure and essential cellular processes and have been employed by fly biologists to understand how tau variants affect neuronal structure and function. Most commonly UAS-mCD8-GFP has been used to label neuronal membranes to study the impact of tau on overall neuronal structure (Williams et al. 2000). To examine the role of tau on different neurobiological processes, responder lines such as UAS-mito-GFP (Pilling et al. 2006) have been used for examining cellular distribution and trafficking of mitochondria in neurons (DuBoff et al. 2012), GFP tagged neuropeptide Y (UAS-PU32M2) has been used for examining axonal transport defects in motor neurons (Mudher et al. 2004), UASsyt-GFP (Robinson et al. 2002) has been used for visualizing synaptic vesicles and terminals (Talmat-Amar et al. 2011), and UAS-LifeAct (Riedl et al. 2008) has been used for labelling F-actin (Frost et al. 2016). In addition to these responder lines there are many other transgenic lines for probing neuronal structure and function (reviewed in Sivanantharajah and Zhang 2015) that have yet to be fully employed in the tauopathy field but may prove to be useful for answering particular questions, especially in the face of newer roles that are now emerging for tau (reviewed in Sotiropoulos et al. 2017). Some examples for labelling cell substructure include: UAS-DenMark for dendritic processes (Nicolaï et al. 2010) and UAS-EB1-GFP for labelling microtubule plus-ends (Rolls et al. 2007). In addition, it is possible to label organelles such as recycling endosomes (UASRab4-RFP) (Sweeney et al. 2006, Zheng et al. 2008), active zones (UAS-BRP-GFP/RFP) (Fouquet et al. 2009), post-synaptic density (UAS-Dlg-GFP) (Koh et al. 1999) or even specific types of neurotransmitter receptors (e.g. UAS-GluRIIA-GFP/RFP for ionotropic glutamate receptors) (Jordán-Álvarez et al. 2012). The availability of these genetic tools and the ease of their use sets the fly apart as one of the most powerful systems for dissecting cellular level processes involved in disease pathogenesis and progression.

Of importance for both identifying and verifying genetic modifiers employable in large-scale enhancer/suppressor genetic screens, for which the fly is particularly suited, there are large collections of responder lines that allow targeted downregulation of specific genes. The available RNAi libraries cover $90 \%$ of the fly genome and allow targeted knockdown of specific genes using the GAL4-UAS system (Dietzl et al. 2007, Ni et al. 2008). UAS-dsRNAi lines are often co-expressed with UAS-tau expressing constructs to knock down genes of interest to study their ability to modify tau mediated phenotypes. Often these vast RNAi libraries are used to screen for tau modifiers, genes that enhance or suppress tau toxicity (Butzlaff et al. 2015). RNAi lines can also be used to validate putative tau modifiers identified by other methods such as microarray analyses or Genome-wide association studies (GWAS) (Dourlen et al. 2017, Shulman et al. 2011, Karsten et al. 2006).

Fortunately for the study of tauopathies in flies, there is a fly orthologue of tau; however, this is not the case for all human disease-associated genes for which GAL4 responder lines exist. For example, there is no homologous protein for alpha-synuclein which is implicated in Parkinson's disease. The case of Amyloid- $\beta$ (A $\beta)$ peptide, which constitutes the plaque pathology in $A D$, is more complicated. Though an orthologous parent protein, $\beta$ amyloid protein precursor-like (APPL), is present in the fly, the $A \beta$ peptides produced by cleavage bare no sequence similarity to human $A \beta$ peptides; however, the ability of fly $A \beta$-like peptides to aggregate and cause age-dependent dysfunction and degenerative phenotypes suggests it may be secondary structure of $A \beta$ peptides rather 
than primary amino acid sequence that determines pathogenesis (Carmine-Simmen et al. 2009). These cases raise interesting questions about the impact of expressing such proteins in fly models of amyloidosis or Parkinson's disease. On the one hand, one may ask whether the fly systems will treat these proteins as "alien proteins" when they are artificially expressed in fly neurons or will they interact in ways comparable to models in which these proteins exist (e.g. as seen with fly $A \beta$ )? On the other hand, one may view these models as ideal platforms for identifying interacting partners without confounding influences of a native orthologue.

\subsubsection{GAL4-driver lines to control where and when tau is expressed}

Stock centres provide thousands of driver lines that can target gene expression in general cell-types (e.g. all neurons, all glia, etc.) to very specific cell-types (e.g. neuronal subtypes such as motor or sensory, neurons using a particular neurotransmitter, etc.). Added to this, the Janelia FlyLight project has generated thousands of driver lines that allow visualization and manipulation of small specific cell-types in the larval and adult nervous systems (Pfeiffer et al. 2008, Jenett et al. 2012). This newer set of driver lines will be an invaluable tool for those seeking to study the effects of disease-associated proteins at the level of different neuronal subsets.

Published studies using fly models of tauopathy have used different strategies to express different variants of tau protein (htau isoforms, bovine tau, fly tau, diseaseassociated variants of htau, etc.) with vastly differing levels of spatial and temporal specificity. These strategies range from global expression models using pan-cellular drivers such as elav-GAL4 and Repo-GAL4 to drive expression in all neurons and all glia, respectively, in the CNS (Colodner and Feany 2010, Wittmann et al. 2001) through to region-based models targeting expression to particular brain structures such as the eye (Jackson et al. 2002) or the mushroom bodies (MB) (Mershin et al. 2004) to tau expression in specific cell-types such as motor neurons (Chee et al. 2005, Mudher et al. 2004), sensory neurons (Sealey et al. 2017, Williams et al. 2000) or neurons using a particular neurotransmitter type (Nishimura et al. 2004, Wu et al. 2013, Wittmann et al. 2001). Table 1 summarises various fly models of tauopathy organised by tissue-level specificity of study (e.g. entire CNS, regional, cell-type specific) and the GAL4 drivers commonly used. Each of these strategies has advantages and disadvantages. Ideally the experimenter knows the limitations of each approach and draws conclusions accordingly. In the next section we provide greater detail about the advantages and limitations of the most commonly used strategies.

\section{The most commonly used Drosophila models of tauopathy: \\ 2.1. Global expression models}

Pan-neuronal expression of tau allows one to understand the central nervous system's response to tau expression. Many studies have driven expression of tau using the pan-neuronal driver, elav-GAL4, and quantified the subsequent effects either: histologically, on overall brain anatomy or particular brain regions (e.g. MB), biochemically, measuring levels and patterns of phosphorylation or protein solubility, or behaviourally, looking for deficits in locomotion or learning and memory (Sealey et al. 2017, DuBoff et al. 2012, Papanikolopoulou et al. 2010, Kosmidis et al. 2010, Folwell et al. 2010, Fulga et al. 2007, Wittmann et al. 2001). Similarly, the role of glia can be tested by targeting tau expression to all glial cells using the driver, Repo-GAL4 (Colodner and Feany 2010), and structural and functional effects of tau in glia can be analysed as they are for neurons. 
Pan-neuronal expression studies of tau-mediated toxicity have played a major role in our current understanding of how tau abnormalities arise. Wittman et al. (2001) were the first to use elav-GAL4 to drive expression of human wildtype (0N4R tau isoform) and mutant tau proteins (R406W) in the fly CNS. These experiments demonstrated the ability of wildtype htau overexpression to cause neurodegeneration and significantly shorten lifespan. Histological analysis of the fly brain following tau expression revealed increased numbers of vacuoles and degenerating cells compared to control brains, but more importantly, the severity of these phenotypes was enhanced by expression of the R406W tau mutant. With this model, the authors also implicated a causal role for hyperphosphorylation in mediating toxicity by showing that tau phosphorylation increased with age in regions of neurodegeneration (i.e. areas near degenerating cells and vacuoles) with no increase in overall tau levels.

Using the same approach, the Skoulakis lab (Papanikolopoulou et al. 2010, Kosmidis et al. 2010) demonstrated that pan-neuronal expression of the tau isoforms 0N4R and 2N4R also caused severe degenerative defects in the MBs with subsequent abolition of olfactory learning. The observed toxicity and dysfunction in the MB were found to be dependent on phosphorylation of tau at two new phosphorylation sites, $\mathrm{Ser}^{238}$ and $\mathrm{Thr}^{245}$, indicating that site-specific phosphorylation rather than general hyperphosphorylation of tau is key. Subsequent work by this group found an added temporal element to tau regulation by phosphorylation showing that sequential phosphorylation at specific sites was essential for the progression of learning defects and premature mortality (Papanikolopoulou and Skoulakis 2015).

Another highly informative observation made by the Skoulakis lab was the finding that tau toxicity and dysfunction are separable (Papanikolopoulou et al. 2010, Kosmidis et al. 2010). Using the TARGET (Temporal and Regional Gene Expression Targeting) system, they supressed tau expression during developmental stages to restrict tau expression to only post-mitotic adult neurons, since elav is expressed early in neuronal development (Berger et al. 2007). Briefly, the TARGET system introduces a temperaturesensitive repressor of GAL4, GAL80 ${ }^{\text {ts }}$, which is expressed in all cells using a tubulin promotor. Shifting incubation of flies between permissive and restrictive temperatures allows regulation of GAL4 mediated transcription. At the non-permissive temperature of $18^{\circ} \mathrm{C}$, GAL80 8 ts suppresses GAL4 function and thus suppresses expression of any UAS responder construct. At $29-30^{\circ} \mathrm{C}, \mathrm{GAL} 80^{\text {ts }}$ is deactivated allowing GAL4 mediated expression to occur. They found that tau expression during development was required to see the morphological degenerative phenotypes in adult MBs with expression of ON4R and 2N4R tau isoforms; however, the lack of tau mediated degeneration, did not translate to a lack of neuronal dysfunction. Although these 0N4R and 2N4R tau-expressing flies had histologically normal MBs, there was still a significant impairment in their performance in learning tasks. This is an important observation demonstrating a clear de-coupling of tau mediated neurodegeneration and neuronal/behavioural dysfunction. It also demonstrates that neuronal dysfunction which precedes neurodegeneration can be significant enough to manifest in a behavioural phenotype. Similar conclusions can be drawn from the findings of other Drosophila models of tauopathy where tau expression was restricted to other neuronal populations (Chee et al. 2005, Mudher et al. 2004). In these studies, htau expression led to quantifiable functional defects but in the absence of overt degeneration.

One general observation in all of these studies is that the severity of phenotypes produced by tau overexpression is dependent on the specific tau variant examined (i.e. 
wildtype isoforms and mutant versions). For example, mutant tau proteins such as R406W show a greater level of toxicity compared to wildtype tau. Even between wildtype tau isoforms, however, there can be variation. For example, pan-neuronal expression of 0N3R tau did not cause MB degeneration, whereas, expression of ON4R and 2N4R tau isoforms did (Papanikolopoulou et al. 2010); a result mirrored by Malmanche et al. (2017) in the fly eye model. Though 0N3R tau seems to have no effect on neuronal degeneration, Sealey et al. (2017) found that expression of ON3R tau caused a reduction in longevity and a faster decline in climbing ability than did ON4R. Conversely, when these two isoforms were compared for their effect on learning and memory, 0N4R tau caused impairment but 0N3R did not. These examples illustrate differences between tau isoforms which can be useful in understanding differing pathology between 3R and 4R tauopathies.

Global expression of tau in glial cells has also been informative in understanding taumediated toxicity. A glial model of tauopathy was created by expressing 0N4R tau in all glial cells (except midline glia) using the Repo-GAL4 driver (Colodner and Feany 2010). Unlike pan-neuronal expression, pan-glial expression of tau during development is lethal; but using the TARGET system to restrict expression to adult glial cells produced phenotypes comparable to pan-neuronal expression, such as reduced lifespan and agedependent cell death. Perhaps the most interesting element of this work was to show the cell non-autonomous effect of tau expression in glia caused death in non-tau expressing neurons, thus illustrating the importance of glial-neuron interactions in disease.

\subsubsection{Cellular mechanisms and toxic tau species in pan-cellular models:}

The mechanisms mediating tau toxicity have been intensely studied in multiple experimental paradigms. The role of phosphorylation and aggregation in these mechanisms is evident from many such studies (Šimić et al. 2016). As described below, Drosophila models of tauopathy have made valuable contributions to our understanding of the complex role of tau phosphorylation and aggregation in pathogenesis, as well as identifying other novel cellular mechanisms of tau toxicity.

One such mechanism involves the actin cytoskeleton. Expression of R406W tau has been shown to cause accumulation of F-actin and the formation of actin-rich rods in degenerating neurons (Fulga et al. 2007), indicating an interaction between the actin microfilament network and the microtubule cytoskeleton that is dependent on normal tau function. A second cellular mechanism is tau-mediated mitochondrial dysfunction. DuBoff et al. (2012) found that expression of R406W tau caused elongation of mitochondria as well as mitochondrial dysfunction and cell death. These phenotypes could be rescued by modulating the expression levels of proteins involved in mitochondrial dynamics (i.e. fission and fusion) such as dynamin-related GTPase (DRP1) and mitochondrial assembly regulatory factor (MARF). Furthermore, expression of E14 tau (a variant of tau that has been pseudophosphorylated by Glu substitution of Ser and Thr at 14 proline-directed kinase target sites) caused greater elongation of mitochondria suggesting that hyperphosphorylation of tau plays a role in the severity of toxicity. A third mechanism of tau toxicity is highlighted by examining the lamin nucleoskeleton, which is a diffuse scaffold composed of lamins, intermediate filament proteins, on which genomic DNA is anchored to the nuclear envelope. Frost et al. (2016) showed that expression of R406W tau resulted in nuclear lamin invagination, tau hyperphosphorylation, activation of the cell cycle, and apoptosis.

Yet another mechanism worth examining is the interaction between tau and other disease-associated proteins such as Amyloid- $\beta(A \beta 42)$ and alpha-synuclein. It was found that co-expression of these proteins enhanced the phenotypes seen when either protein 
was expressed alone in both locomotor and longevity assays (Folwell et al. 2010, Roy and Jackson 2014). The tau/Ab42 exacerbation phenotypes were rescued following treatment with $\mathrm{LiCl}$, an inhibitor of GSK-3 $\beta$ /shaggy (sgg), indicating that phosphorylation plays a role in the interaction between tau and $A \beta$.

Pan-cellular expression experiments in fly have also been informative in demonstrating the complex relationship between tau toxicity and tangle formation. In their first study, Wittman et al. (2001) demonstrated that tau-mediated degeneration following pan-neuronal tau expression does not require tau aggregation into tangles. This is also supported by other studies that restricted tau expression to specific neurons types, such as sensory or motor neurons (Williams et al. 2000, Mudher et al. 2004). Like Wittman et al. (2001), these studies also reported tau-mediated phenotypes in the absence of overt tau tangles, further dissociating tau-toxicity and tangle formation. Adding to this argument, Cowan et al. (2015) found that rescue from tau dysfunction resulted in formation of nontoxic, insoluble tau oligomers. This implies that like tangles, insoluble tau oligomers are also not required for tau-mediated dysfunction or degeneration. This leaves the possibility that soluble tau oligomers, rather than larger insoluble tau aggregates are the toxic species in tauopathies (reviewed in Cowan and Mudher 2013) and should be targeted by diseasemodifying therapeutic interventions. Indeed, some studies have demonstrated appearance of oligomeric tau species at various time-points correlating to disease progression (Wu et al. 2013, Ali et al. 2012). In sharp contrast, pan-glial expression of tau does result in the formation of fibrillary tangles in glial cells (Colodner and Feany 2010). This demonstrates that it is not necessarily the shorter lifespan of Drosophila that is responsible for the lack of tau aggregation in most fly models and that other, possibly tissue dependent factors, play a role in this pathological process. An interesting observation in this study was that switching off tau expression in 20-day old flies for 10 days caused a reduction in observed cell death; but this reduction in tau-mediated cell death was not correlated with a reduction in total tangle number. This serves to further underline the dissociation between tau toxicity and tau tangles, at least in Drosophila models of tauopathy.

\subsection{Targeting specific brain structures: the eye and mushroom bodies \\ 2.2.1 Targeting tau to the fly eye}

Greater cellular resolution can be achieved by restricting tau expression to specific brain structures, such as the fly eye and mushroom bodies. Using eye-specific GAL4 drivers, expression of tau in the eye produces a "rough eye" phenotype which is reflective of retinal cell degeneration. This is most commonly done using the GMR-GAL4 driver line, but rough eyes can also be generated using sev-GAL4 (a more selective eye driver) as well as elav-GAL4. The eye is used as a target because it provides a clearly visible phenotype that is easy to define and quantify, thus making it amenable to high throughput

enhancer/suppressor screens. Such screens have enabled the unbiased identification of novel modifiers of disease-associated proteins and pathways involved in disease processes. For example, Butzlaff et al. (2015) screened for modifiers of R406W tauinduced toxicity by examining more than 7000 RNAi lines which covered more than half of all protein coding fly genes and equivalent to over $90 \%$ of the fly genes known to have human orthologs. The screen identified 62 genes that, when silenced, modified tau-induced toxicity. Standout genes identified were three subunits of the Dynein/Dynactin complex, a key part of the axonal cytoskeleton and retrograde axonal transport mechanism (Kardon and Vale 2009). The validity of these candidates was tested using larval motor neurons showing that pan neural R406W tau expression coupled with the silencing of 
Dynein/Dynactin complex members had strong pathological effects in the axon, inhibiting retrograde but not anterograde axonal transport with only minor changes at synapses. This work suggests that an early step in the pathogenic process is axon degeneration.

Ambegaokar and Jackson (2011) used the eye for a functional genetic screen using loss-of-function and gain-of-function alleles to identify modifiers of tau-induced neurotoxicity using the 2N4R tau isoform. This study identified a wide range of modifiers that included some well-known tau kinases including GSK-3ß/sgg, PAR-1/MARK, CamKI and Mekk1. A large proportion of the identified genes included genes involved in diverse cellular functions such as: autophagy, cell-cycle, RNA-associated proteins and chromatin-binding proteins. Smaller numbers of identified genes included mitochondrial proteins, lipid trafficking, golgi proteins, kinesins and dynein and the Hsp70/Hsp90-organizing protein (Hop). The work opened the way for complex network analysis to identify regulatory networks and suggested several other genes highly associated with these functional modifiers, including genes related to the PI3K, Notch, BMP/ TGF-b and Hedgehog pathways, and nuclear trafficking. This study serves to illustrate the power of the eye as a platform for such large-scale screens and the ability to open up completely new avenues of investigation.

In the context of the outcomes of a screen, the fly eye also provides a platform for more directed approaches to study novel putative tau phenotype modifiers, be they identified through such enhancer/suppressor screens as above, or from microarray analyses or human GWAS studies (Karsten et al. 2006, Dourlen et al. 2017, Shulman et al. 2011). The approach is exemplified by the work of Dourlen et al. (2017) who took 19 loci identified in a GWAS meta-analysis for AD and used Drosophila to screen constructs targeting orthologs of candidate risk genes within these loci for their ability to modify tau neurotoxicity. They identified several tau toxicity modulators including: Drosophila Amph (ortholog of BIN1), p130CAS (CASS4), Eph (EPHA1), Fak (PTK2B) and Rab3-GEF (MADD). Using the fly eye to validate the outcomes of such screens is still in its infancy but it is clear that the fly will become an important tool in the validation of putative interacting genes. Furthermore, the high throughput nature of this type of screening could also lend itself to large scale drug screening. In this light, the fly provides the perfect organism to do "proof-of-principle" studies, because one can test hypotheses in large scale and in a short span of time before attempting targeted smaller-scale similar studies in models that are more expensive and laborious to use.

Beyond genetic screens, the fly eye has also been informative for dissociating tau mediated toxicity and dysfunction and the role of tau phosphorylation in each. Chouhan et al. (2016) found that expression of htau using Rh1-GAL4, a driver that is turned on after photoreceptor maturation, resulted in no degeneration of the fly retina; in fact, experimental and control retinas were indistinguishable. However, ultrastructural analysis found some disruption of photoreceptors and synaptic organization and a functional analysis of the retina using electroretinograms (ERG) showed that htau expressing flies had a significant loss in ERG responses, most likely due to photoreceptor dysfunction. This observation is further informed by the work of Malmanche et al. (2017) who showed that developmental effects of tau were isoform specific. Where the $4 R$ taus (2N4R and 0N4R) induced a rough eye phenotype when driven by GMR-GAL4, ON3R did not. Together, these studies demonstrate an important division between tau-mediated degeneration versus dysfunction.

\subsubsection{Cellular mechanisms for tau toxicity and dysfunction in the eye model}


The fly eye is not just of value in wide-scale screens and functional testing it has also contributed enormously as an experimental platform to dissect cellular mechanisms of tau induced degeneration. One well-studied mechanism is phosphorylation of tau and the role of putative tau kinases and impact of differential site-specific phosphorylation. Abnormal tau hyperphosphorylation has traditionally been implicated in mediating tau-loss-ofmicrotubule binding function effects (lqbal et al. 2008, Grundke-lqbal et al. 1986), and as described here and in later sections of this review, studies in Drosophila have provided further support for this hypothesis (Quarishe et al. 2013, Cowan et al. 2010b, Quraishe et al. 2017). Additionally, evidence from Drosophila models has also shed light on the role of tau phosphorylation in neurodegeneration and its potentially complicated role in influencing tau aggregation. That hyperphosphorylation accompanies neuronal dysfunction and neurodegeneration has been demonstrated by many groups (Wittmann et al. 2001, Mudher et al. 2004, Sealey et al. 2017 and others). However, the causal role of site-specific phosphorylation in differentially contributing to neuronal dysfunction (via reduced microtubule binding) and neurodegeneration has come from Drosophila models employing phospho-mimicking or non-phosphorylatable tau mutants expressed in the fly eye.

Pioneering these studies, Nishimura et al. (2004) showed that the Drosophila PAR-1 kinase can initiate tau toxicity by triggering a temporally ordered phosphorylation of tau, directly phosphorylating tau at $\operatorname{Ser}^{262}$ and $\operatorname{Ser}^{356}$. This phosphorylation event is a prerequisite for the activity of downstream kinases, such as GSK-3ß/sgg and Cdk5, to phosphorylate other sites and generate disease-associated phospho-epitopes. This was a powerful study that began to dissect the effects of phosphorylation on tau toxicity and opened the door to new potential routes to therapeutic targets. Following on from this, the work of Chatterjee et al. (2009) found that resistance of tau to phosphorylation by GSK$3 \beta /$ sgg caused substantially greater toxicity due to greater affinity for microtubule binding than wildtype tau. Together these studies suggest that tau phosphorylation at the PAR1/MARK sites (e.g. Ser ${ }^{262}$ and $\mathrm{Ser}^{356}$ ) cause neurodegeneration while tau phosphorylated at the GSK-3 $3 /$ sgg (e.g. Ser ${ }^{202}$, Thr ${ }^{205}$, $\mathrm{Thr}^{212}$, Ser ${ }^{214}$, $\mathrm{Thr}^{231}, \mathrm{Ser}^{396}, \mathrm{Ser}^{404}$ ) cause dysfunction, possibly via microtubule binding effects.

A second mechanism of toxicity that has emerged from studies conducted in the fly eye is autophagy, an important element of normal cell physiology that is implicated in a number of neurogenerative disorders. Bakhoum et al. (2014) used the fly eye to show that autophagy could play a role in tauopathies. They found that misexpression of htau induced accumulation of autophagic intermediates with large vacuoles, termed giant autophagic bodies (GABs), which are indicative of autophagic dysfunction. Furthermore, inducing autophagy could rescue the tauopathy phenotype, suggesting that formation of GABs may be a compensatory mechanism rather than a trigger of neurodegeneration and that disruption of autophagic processes may play a key role in the progression of tau pathology.

A third mechanism is protein glycosylation, which has been reported to be altered in AD brains (Butterfield and Owen 2011, Kanninen et al. 2004, Schedin-Weiss et al. 2014, Zhu et al. 2014). The role that protein glycosylation plays in AD pathology is largely unknown. To begin to unravel glycosylation as a possible mechanism of the degenerative process, Frenkel-Pinter et al. (2017) used tau overexpression in the fly eye as the basis to analyse the effects of reducing the expression of fly homologs of glycosylation-related genes identified from a bioinformatics analysis of human studies. They identified glycosylation genes that can either augment or ameliorate the tauopathy phenotypes suggesting that Ost<Delta>, I(2)not and $\beta-4$ GalT7 are suppressors of tau-mediated toxicity 
and pgnat5 and CG33303 are enhancers. These novel results suggest that specific alterations in protein glycosylation may play a causal role in the progression of $A D$, and present potential therapeutic targets for treatment of disease.

\subsubsection{Targeting tau to mushroom bodies}

The mushroom body is the conserved brain centre for learning and memory in insects, and as such, can be argued as functionally analogous to the human hippocampus. Structurally it is made up of glia and three major classes of neurons (Lee et al. 1999). Manipulating tau expression in mushroom bodies has been essential in demonstrating a conserved role of tau in learning and memory in flies. Assessing the impact of tau on mushroom bodies has been done in two ways: by examining their structure and function upon expression of tau using pan-neuronal drivers such as elav-GAL4 (see section 2.1) or late onset drivers such as c492 and c772. Restriction of tau expression to post-mitotic neurons in the mushroom bodies has been key in detangling tau-mediated toxicity from taumediated dysfunction. The results of using the TARGET system to limit elav-GAL4 driven tau expression to adult mushroom bodies are discussed above. When driven by elav-GAL4, tau has an effect on mushroom body structure and function; however, every neuron in the central nervous system has also been exposed to insult by tau expression so one needs to appreciate that the behavioural output from such studies may reflect the collective impact of tau on a number of circuits. Use of post-mitotic drivers that are spatially restricted to MBs provide greater cellular resolution and have revealed similar results. Mershin et al. (2004) saw an effect on associative olfactory learning and memory upon expression of tau proteins in MB neurons, but olfactory conditioning-relevant osmotactic and mechanosensory responses were not affected. These behavioural phenotypes were observed without overt neurodegeneration of MB neurons or any effect on longevity. These studies once again stress that tau-mediated dysfunction can manifest in behavioural phenotypes prior to onset of neurodegeneration or in fact tangle formation.

\subsection{Targeting selective subtypes of neurons: motor, sensory, neurotransmitters}

Targeting expression of tau to single or small numbers of neurons provides a tool to begin to understand the mechanisms of tau mediated toxicity and dysfunction at single cell resolution and gain insights into the cellular mechanism that underlie these processes. Typically, this approach has targeted expression of tau to specific neuronal subtypes such as sensory and motor neurons or neurons that use a particular neurotransmitter. In the first study examining the impact of htau in a fly model, Williams et al. (2000) expressed tau using c161-GAL4, which targets expression specifically to proprioceptive sensory neurons, and uncovered several indicators of neurodegeneration: axon loss, axon defasciculation, reduced arborization and axonal swellings. The work also reported that phenotypic severity increased with age, as would be expected of an age-dependent neurodegenerative process. Sensory neurons have also been used to understand isoform specific differences. Sealey et al. (2017) compared toxicity of ON3R and ON4R taus in sensory neurons in the fly eye using R7-GAL4 and found greater degeneration of neurons with ON4R at 20 days than with ON3R tau. This work demonstrated a key difference in the behaviour of two wildtype tau isoforms in that $0 \mathrm{~N} 4 \mathrm{R}$ tau causes greater overall toxicity to neurons than ON3R tau.

A more commonly used approach in the field is to drive expression in larval motor neurons, using drivers such as D42-GAL4 and OK6-GAL4, and visualize activity within readily accessible larval motor neuron axons and the neuromuscular junction (NMJ) using assays for axonal transport and synaptic function, respectively. The larval NMJ is a widely used system to analyse the mechanisms of synapse formation, growth and maintenance 
(Menon et al. 2013, Keshishian et al. 1996). This model affords numerous advantages in that it allows analysis of the effects of tau expression with subcellular resolution, meaning that one can quantify effects on clearly visible individual synaptic boutons (Chee et al. 2005) and examine axon transport defects along well-defined peripheral nerves in real time and in living intact animals (Mudher et al. 2004). Most importantly, this system is amenable to electrophysiology, which allows direct measurement of neuronal function (Chee et al. 2005). Using this model, it was shown that some of the earliest consequences of tau expression is breakdown of the axonal cytoskeleton and the disruption of axon transport leading to the aggregation of transport vesicles (Mudher et al. 2004). As a result of these transport defects there is a lack of functional mitochondria at the NMJ causing synaptic dysfunction and compromised synaptic vesicle cycling causing a diminution of synaptic transmission (Chee et al. 2005). This system has also been useful in demonstrating isoform dependent differences in tau-mediated dysfunction. Sealey et al. (2017) found that ON3R tau but not ON4R tau caused locomotor dysfunction and impaired axon transport, again demonstrating an association of $4 \mathrm{R}$ isoforms are with greater toxicity and $3 \mathrm{R}$ isoforms with greater dysfunction.

Using the same system as background and using both axon transport and locomotor phenotypes caused by tau expression, Mudher et al. (2004) demonstrated that expressed htau is abnormally phosphorylated. Co-expression of an activated GSK-3 $\beta$ exacerbated the tau phenotypes and conversely, inactivation of GSK-3 $\beta$ by lithium chloride (a GSK-3 $\beta$ inhibitor) reversed them. This suggested that the tau induced phenotypes are regulated by tau phosphorylation. Subsequent work with this model showed that the mechanism by which htau mediates its toxic effects is twofold: first, highly phosphorylated tau has reduced binding to microtubules, and second, it sequesters dtau from microtubules, implicating both a toxic-loss-of function and toxic-gain-of function in the context of microtubule binding. A consequence of this is disruption of the microtubular cytoskeleton, resulting in a reduction in the number of intact, correctly-aligned microtubules and incorrectly oriented microtubules. These effects were phosphorylation dependent ass suppression of tau phosphorylation increased microtubule binding of both htau and dtau and restored cytoskeletal integrity (Cowan et al. 2010b). These results are further supported by Talmat-Amar et al. (2011), who found that AP tau (a variant of tau that has been hypophosphorylated by Ala substitution of Ser and Thr at 14 proline-directed kinase target sites) had a greater affinity for microtubules than htau or E14 tau and caused significant axon transport defects when expressed in larval motor neurons. Together with studies from the eye model, it is clear that excessive (as described by Chatterjee et al. 2009, Talmat-Amar et al. 2011) and sub-optimal (as described by Mudher et al. 2004, Cowan et al. 2010b) microtubule binding is detrimental.

In addition to the influence of phosphorylation on microtubule binding, some theories propose that it stimulates tau aggregation and therefore may have a two-fold mechanism of toxicity. However, in the studies of Mudher et al. (2004) and Cowan et al. (2010b) there was no evidence of hyperphosphorylation causing tau aggregation. This demonstrates that hyperphosphorylation induced loss-of-microtubule binding function is sufficient to cause neuronal dysfunction and behavioural deficits, and these phenotypes are independent of tau aggregation. Subsequent studies demonstrated that microtubule stabilising agents rescued these phenotypes and intriguingly did so without reducing tau phosphorylation (Quraishe et al. 2016). This highlights the critical role played by loss of tau's microtubule-binding function in mediating neuronal dysfunction in early (pre-tau aggregation) stages of disease. 
Other experiments have chosen to focus on the effect of targeting tau to neuron subtypes on the basis of their primary neurotransmitter system, with a focus on the cholinergic system which is implicated in AD (Sims et al. 1983). Wittman et al. (2001) used Cha-GAL4 to examine the effect of htau on cholinergic neurons in the central complex of adult flies and found evidence of neurodegeneration without any subsequent effect on longevity. Nishimura et al. (2004) used the same driver to show a greater amount of phosphorylation at two sites, $S^{262}$ and $S^{356}$, in the microtubule binding domain of htau in cholinergic nerve terminals in the optic medulla when PAR1 kinase and htau were coexpressed; levels higher than when either were expressed alone. They also noted neurodegeneration (e.g. vacuole formation) in cell bodies and processes in the giant interneuron commissure with co-expression of PAR1 and h-tau. These effects of tau are likely to result from the presence or absence of molecular factors such as tau kinases or tau phosphatases in these neurons rather than a preferential susceptibility of cholinergic neurons to tau-toxicity.

Expanding on tissue-specific susceptibility, Wu et al. (2013) compared the effect of tau expression on a wider range of neurotransmitter-specific cell-types, including dopamine, GABA, serotonin and glutamate. They showed that expression of tau in dopaminergic, serotonergic and some peptidergic neurons produces a marked decrease in the number of those neuron types in the adult brain. In contrast, expression of tau in glutamatergic and GABAergic neurons did not produce any loss of neurons. Importantly, this was one of few studies to have demonstrated bona-fide tau aggregates in Drosophila CNS neurons. Tau oligomers, filaments and tangle-like structures were demonstrated when tau was expressed in dopaminergic neurons using a number of techniques (biochemical, ultrastructural and immunohistochemical). Here too, one may argue the susceptibility of the neuronal populations that succumbed to tau-mediated degeneration may arise due to factors promoting tau phosphorylation and aggregation. Further studies are needed to compare the tau kinase/phosphatase profiles of susceptible and resistant cell-types, as well as their endogenous protein clearance capabilities to understand how they confer vulnerability or resistance to tau-mediated degeneration. This is one area of research that can benefit enormously from the superior genetic tractability of Drosophila, that can enable such studies more easily than in other models of tauopathy.

\section{Considerations for commonly used Drosophila models of tauopathy}

\subsection{Controlling for expression levels of responder lines in comparative studies:}

As with studies on all animal systems, many of the foundational tools used to study neurodegeneration in Drosophila have some limitations in the spatial and temporal aspects of disease-gene expression. However, the field is constantly evolving and developing new genetic tools and knowledge that allow us to undertake more precisely defined experiments. For example, gaining better control of expression levels of tau transgenes across studies to help comparative studies of tau isoforms, tau mutants, or different disease-causing proteins. It is arguable that though this limitation of disease-gene expression affects all model systems, the genetic tractability of flies makes them the most amenable to overcoming such limitations than any other model system. The firstgeneration responder lines were typically inserted randomly into the fly genome and expression levels of different proteins were not always same as the result of genomic position effects on transcriptional levels, even when using the same GAL4 driver. To bring better consistency to comparative experiments and reduce expression level differences, 
tools have been developed that can minimise these differences. For example, using fly lines in which transgenes are inserted into the same genomic location to reduce site-dependent effects on expression levels as done by Fernius et al. (2017), who showed that when inserted in same genomic location expression of all six hTau isoforms exhibited comparable toxicity in flies. Alternatively, it is possible to use a more natural expression system by replacing the endogenous dtau coding region with the htau coding sequence (Gorsky et al. 2017). This has a two-fold impact by eliminating endogenous tau expression, and by driving htau expression at normal physiological levels. Using this approach Gorsky et al. (2017) found that although naturally expressed htau had no effects on the morphology or physiology of expressing neurons, it did ameliorate an Amyloid- $\beta$ phenotype and acetylation at specific tau residues supressed phosphorylation and microtubule binding. The importance of this lies in the ability to distinguish between tau functions and modifications evident under normal physiological conditions from scenarios when it is overexpressed.

\subsection{Developmental expression of tau}

One concern with some commonly used Drosophila tauopathy models is that the ensuing phenotypes could be developmental in nature. Pan-neural drivers such as elavGAL4 are expressed during early neural development, including in neuronal precursor cells. Similarly, some eye-specific drivers such as GMR-GAL4 and sev-GAL4 are expressed during development in cells of the differentiating eye discs, and in the case of GMR-Gal4 in non-neuronal cells such as glia (Freeman 1996, Tomlinson et al. 1987). There is evidence that developmental expression of tau can have detrimental effects since driving a tau-lacZ transgene in rapidly dividing cells using an engrailed-GAL4 driver interferes with mitosis and can cause lethality (Phelps and Brand 1998). This implies that one must interpret the tau phenotypes obtained using drivers that may drive tau expression developmentally with care. It is important in those models to ascertain whether the neurodegeneration and consequent phenotypes reported reflect an age-related pathogenic process (i.e. truly modelling age-related disease pathogenesis) or arise due to developmental effects of tau expression.

This concern has been addressed by most groups in two ways. First, by selecting to study tau phenotypes that are adult onset in nature, where there is no tau phenotype on emergence as an adult and that which develops with increasing age (e.g. Sealey et al. 2017). Or second, by restricting transgene expression to post-mitotic adult neurons (e.g. Papanikolopoulou et al. 2010, Kosmidis et al. 2010, Chouhan et al. 2016 and others). Temporal restriction of tau expression can be achieved by using post-mitotic neuronal drivers such as APPL-GAL4 for pan-neuronal expression, Rh1-GAL4 in photoreceptors and c492-GAL4 and c7772-GAL4 in mushroom bodies. Alternatively, one could determine the expression pattern of the lines of choice in developmental stages before embarking on studies with a novel GAL4 line. If suitable GAL4 drivers cannot be found, tools exist to experimentally restrict expression to post-mitotic neurons using inducible systems such as TARGET or GeneSwitch (Osterwalder et al. 2001, McGuire et al. 2004), both of which provide an experimentally controlled temporal switch for expression.

\subsection{Cellular resolution and the heterogeneity of tau processing}

One facet of fly models shared by all disease models including rodents is a lack of cellular resolution, especially when using pan-neural GAL4 drivers to drive tau expression. This can serve both as a limitation and a strength. It can be a limitation if one wanted to understand what underpins region-specific neuronal vulnerability to explain regional selectivity in different tauopathies. Alternatively, it may be regarded as a strength if one 
were to view some tauopathies, like AD, as "systems" diseases, where neuronal connectivity underpins spatio-temporal regional spread of disease. Global expression experiments give a valuable general impression of how the nervous system collectively responds to an insult by toxic protein expression. However, they cannot give nuanced information about cell-specific vulnerability of thousands of different neuronal cell-types in the brain.

Better spatial resolution of targeted tau expression has been achieved in Drosophila studies using brain-region specific drivers (e.g. mushroom body drivers, retinal drivers, motor neuron drivers, etc.). By targeting the eye and mushroom bodies, there is improved resolution in that tau is targeted to specific brain regions; however, one must also consider whether expression in non-neuronal tissues such as glia contributes to the phenotype with those drivers. In the case of mushroom bodies, which are a heterogeneous cellular structure in which different neuronal subtypes are targeted together, one assumes here that all these neuron subtypes respond equally to tau expression. Larval motor neurons, on the other hand may provide single cell resolution and in vivo imaging capabilities but are limited in not enabling assessment of age-dependent phenotypes. However, this can be overcome by using assays employed in other arenas of Drosophila neurobiology which use adult specific drivers with enhanced in vivo imaging capabilities (e.g. wing and leg assays) (Vagnoni and Bullock 2016).

Another important consideration is that one cannot always extrapolate results obtained from one specific population of neurons to generalise for the entire brain. Some studies in Drosophila have shown cell-type specific differences in tau biochemistry. Grammenoudi et al. (2006) found that tau proteins showed different phosphorylation patterns and stability when expressed in different neuron subtypes, including photoreceptors, mushroom body neurons, and motor neurons. This suggests that different neurons handle tau in different ways and that extrapolating results from one neuron type to another does not always paint the full picture. This is supported by the conspicuous lack of histologically evident tau aggregates upon tau expression in all CNS tissues in Drosophila except dopaminergic neurons (Wu et al. 2013) or glial cells (Colodner and Feany 2010). Given the observation that different neurodegenerative diseases show clear associations with specific brain regions, which in part is due to selective vulnerability of neurons to toxic proteins, it is important to target smaller subtypes of neurons, or even single-cells in order to understand the details of the degenerative process. The tools to do this already exist for Drosophila. Spatial restriction of tau expression can be achieved by exploiting the rapidly expanding array of GAL4-driver lines that offer more precisely targeted and well documented expression patterns. These lines allow single-cell or cell-type specific resolution by using GAL4 driver lines that restrict expression to small groups of neurons. This has become possible using intersectional strategies such as the Split-GAL4 system (Luan et al. 2006) to generate lines that drive Gal4 expression in small, specific classes of neurons (Jenett et al. 2012, Pfeiffer et al. 2010). These lines have yet to be fully applied to the study of neurodegenerative disease in flies but provide excellent potential for understanding specific elements of the disease process at single cell resolution.

\section{Summaries and conclusions:}

\subsection{Key contributions to the field by the fly}

Collectively, the work presented in this review provide different perspectives of the disease mechanisms with each study providing valuable advances in knowledge. When 
the discoveries made across different fly models are consolidated, a common story begins to emerge that provides insight into the causes of tau abnormalities, the role of these abnormalities in mediating tau-mediated toxicity and/or dysfunction and the nature of causative species mediating tau-toxicity.

It is traditionally believed that tau becomes pathogenic in tauopathies because it becomes abnormally hyperphosphorylation, misfolded and aggregated. Drosophila models of tauopathy have contributed to our understanding of the role of hyperphosphorylation in tau toxicity. Though kinases and phosphatases known to cause hyperphosphorylation had been identified in other experimental paradigms (reviewed in Dolan and Johnson 2010), the Drosophila models of tauopathy have identified a number of novel tau kinases including components of the JAK/STAT signalling pathway (Colodner and Feany 2010), and Nuak1, an AMP-activated protein kinase (AMPK)-related kinase (Lasagna-Reeves et al. 2016) amongst others. Furthermore, the role of known tau kinases in mediating tau phosphorylation and the pathological consequences downstream have been further elaborated in Drosophila. For example, GSK-3 $\beta / \mathrm{sgg}$ phosphorylates tau at the same epitopes as in rodents and humans, but studies exploring the impact of this kinase on tau in the fly have been important in dissecting the specific tau phosphorylation sites associated with dysfunction (e.g. microtubule destabilisation) and those associated with toxicity (e.g. degeneration) (Nishimura et al. 2004, Chatterjee et al. 2009). This point stresses the importance of integrating work from rodents and flies. Additionally, fly work on tau phosphorylation has demonstrated that tau phosphorylation in vivo is multi-faceted - it can occur in a sequential manner dictated by previous phosphorylation events (Chatterjee et al. 2009) and the pattern of phosphorylation is tissue and age dependent (Grammenoudi et al. 2006, Papanikolopoulou and Skoulakis 2015). How tau phosphorylation influences aggregation is not entirely clear, though all Drosophila data imply that the relationship is not straight-forward, because while hyperphosphorylation is evident in all models, aggregation is not. Indeed, in one model aggregation into tau oligomers was associated with rescue of neuronal dysfunction (Cowan et al. 2015).

The genetic tractability of Drosophila lends itself to unbiased enhancer/suppressor screens, which have revealed a number of novel cellular mechanisms that may prove useful in understanding the wider disease process. This includes, but is not limited to, tau phosphorylation by newly characterized kinases such as Nuak1 (Lasagna-Reeves et al. 2016), the importance of the axonal cytoskeleton and retrograde axonal transport (Butzlaff et al. 2015), and the role of autophagy, RNA processing, protein translation and ribosomalassociated genes (Ambegaokar and Jackson 2011). Many potential mechanisms have also been identified from hypothesis-driven studies which have implicated the actin cytoskeleton, the lamin nucleoskeleton, mitochondrial dysfunction, axon trafficking, and synaptic dysfunction in tau toxicity. Understanding the root cause of tau toxicity on these cellular processes may present focus areas for targeted drug development in disease-modifying therapies. Similarly, the genetic tractability of the fly model enables it to serve as a platform to study the role of targets identified from human GWAS studies. This will allow the dissection of newly identified disease mechanisms on a larger scale; arguably, this cannot be done, at this level, in any other model organism.

Another contribution is the appreciation that tau mediated dysfunction and/toxicity is tissue and isoform dependent. Drosophila models illustrate that the impact of pathological tau depends heavily on how a particular tau isoform is handled by a tissue. Some tissues are resistant to neurodegeneration whilst others are vulnerable; some phosphorylation 
profiles, aggregation propensities and even dysfunction versus degeneration phenotypes are evident when certain tau isoforms are expressed but not others (Sealey et al. 2017, Papanikolopoulou et al. 2010, Kosmidis et al. 2010). Clearly a "one-size-fits-all" scenario does not explain how the six tau isoforms cause dysfunction and degeneration in tauopathies. This conclusion can be drawn largely because of what we have learnt from fly models of tauopathy, and it is reassuring that rodent models support this idea (McMillan et al. 2008).

Lastly, the fly model has been important for illustrating that tau-mediated neurodegeneration and dysfunction can occur without the formation of neurofibrillary tangles. This suggests that aggregation and tangle formation during AD is likely to be a late or end stage process or one that is specific to cell-type. It is possible that if flies lived longer, they may accumulate toxic tangles, though this seems unlikely because complete degeneration of neurons is often seen without tangle formation. However, there are some cells in which tangles are observed: glial cells and dopaminergic neurons. Therefore, a more likely solution is that cells resistant to tangle formation lack some molecular component that facilitates or promotes tau aggregation; whereas, the cellular environment of cells like glial cells and dopaminergic cells may be more conducive to tau aggregation because such molecular components are more abundant. Regardless, induction of taumediated toxicity and dysfunction without tangles suggests that the toxic species of relevance is really another form of tau, likely soluble, hyperphosphorylation tau. That this toxic tau species may be a small soluble aggregate is suggested by a very interesting observation by Passarella et al. (2018) who reported a conspicuous absence of taumediated degeneration and behavioural phenotypes in transgenic flies expressing a nonaggregate prone htau mutant. This hypothesis is not without some corroboration in mammals with studies in mice that have shown that tau-mediated dysfunction occurs in the absence of neurofibrillary tangle formation and cell death (Kimura et al. 2007), and that targeting tau oligomers reverses tauopathy phenotypes in a rodent model of tauopathy (Castillo-Carranza et al. 2014).

\subsection{Conclusions}

The last two decades has established the fly as a useful animal model to gain rich new insights into human neurodegenerative diseases. The tools for use on Drosophila are constantly advancing and improving our ability to study human diseases in the fly with greater resolution. The combination of precisely controlled disease related transgene expression with new reporter lines that allow us to analyse the different functional compartments and components of neurons (e.g. cytoskeleton, axon transport, synaptic function and organelles, etc.) provides an unparalleled array of tools that can be deployed to analyse neurodegenerative processes in the fly. With this will come greater experimental insights into disease processes, making the fly the most powerful, non-mammalian in vivo system for studying the mechanisms of neurodegeneration.

\section{Acknowledgements}

We apologize to all authors whose work could not be cited in this article due to the limitations of space.

\section{Funding}

This work was supported by the Alzheimer's Society UK [grant number 315 (AS-JF-16004)]. 
Table 1. Summary of commonly used Drosophila models of tauopathy

\begin{tabular}{|c|c|c|c|}
\hline $\begin{array}{c}\text { Tissue level } \\
\text { resolution of model }^{*}\end{array}$ & $\begin{array}{c}\text { GAL4 } \\
\text { Drivers }\end{array}$ & Advantages & Considerations \\
\hline $\begin{array}{l}\text { Entire CNS } \\
\text { a. Pan-neuronal }\end{array}$ & elav & $\begin{array}{l}\text { (i) Observe tau-mediated toxicity } \\
\text { (e.g.neurodegeneration, shortened } \\
\text { lifespan) and dysfunction (e.g. climbing } \\
\text { defects) (Wittmann et al. 2001) }\end{array}$ & $\begin{array}{l}\text { (i) Expression during early neuronal } \\
\text { development; noted degeneration due to } \\
\text { developmental tau expression } \\
\text { (ii) Expression in non-neuronal cells (e.g. } \\
\text { embryonic glia and glioblasts (Berger et al. } \\
\text { 2007)) } \\
\text { (iii) Expression in many neuron cell-types }\end{array}$ \\
\hline b. Pan-glial & Repo & $\begin{array}{l}\text { (i) Can recapitulate disease related } \\
\text { phenotypes such as tangle formation } \\
\text { (Colodner and Feany 2010) } \\
\text { (ii) Can study cell non-autonomous effect } \\
\text { of glial tau on neurons }\end{array}$ & $\begin{array}{l}\text { (i) Not specific to glial cell-type (expression in all } \\
\text { glial types except mid-line glia) }\end{array}$ \\
\hline $\begin{array}{l}\text { REGIONAL } \\
\text { a. Eye model }\end{array}$ & $\begin{array}{l}\text { GMR, sev, } \\
\text { Rh1, R7 }\end{array}$ & $\begin{array}{l}\text { (i) Well established assays, easy visual } \\
\text { phenotype to qualify and quantify } \\
\text { (ii) Highly amenable to large scale } \\
\text { modifier/suppressor screens to identify } \\
\text { tau modifiers (Butzlaff et al. 2015, } \\
\text { Ambegaokar and Jackson 2011, Dourlen } \\
\text { et al. 2017, Shulman et al. 2011). } \\
\text { (iii) Observe tau-mediated toxicity (e.g. } \\
\text { neurodegeneration) and dysfunction (e.g. } \\
\text { reduction in retinal function (Chouhan et } \\
\text { al. 2016)) } \\
\text { (iv) Available GAL4 drivers to restrict } \\
\text { expression to post-mitotic adult neurons } \\
\text { (e.g. Rh1)(Chouhan et al. 2016) }\end{array}$ & $\begin{array}{l}\text { (i) GMR drives expression during photoreceptor } \\
\text { development; noted degeneration due to } \\
\text { developmental tau expression } \\
\text { (ii) Not all driver lines are specific to one cell- } \\
\text { type (e.g. GMR drives expression in glia and } \\
\text { many neuron cell-types) }\end{array}$ \\
\hline
\end{tabular}




\begin{tabular}{|c|c|c|c|}
\hline & & $\begin{array}{l}\text { (v) Available GAL4 drivers to spatially } \\
\text { restrict expression to specific cell-types } \\
\text { (e.g. Rh1, R7 and sev) }\end{array}$ & \\
\hline $\begin{array}{l}\text { b. Mushroom bodies } \\
\text { (MB) }\end{array}$ & $\begin{array}{l}\text { elav, c492, } \\
\text { c772 }\end{array}$ & $\begin{array}{l}\text { (i) Can target MBs to study effects of tau } \\
\text { on learning and memory in adult flies } \\
\text { (Mershin et al. 2004) } \\
\text { (ii) Driver lines that express only in adult } \\
\text { post-mitotic cells of MB are available } \\
\text { (e.g. c492, c772) (Mershin et al. 2004) } \\
\text { (iii) Observe tau-mediate } \\
\text { neurodegeneration and dysfunction }\end{array}$ & $\begin{array}{l}\text { (i) Drivers such as elav are global and not } \\
\text { specific to just the MBs, meaning one cannot } \\
\text { rule out the effects of tau expression on other } \\
\text { brain regions/circuits } \\
\text { (ii) Some drivers induce expression during early } \\
\text { neuronal development (e.g. elav); noted } \\
\text { degeneration due to developmental tau } \\
\text { expression }\end{array}$ \\
\hline $\begin{array}{l}\text { CELL-TYPE SPECIFIC } \\
\text { a. Motor-neurons }\end{array}$ & D42, OK6 & $\begin{array}{l}\text { (i) Larval neuromuscular junction is a } \\
\text { well-established model } \\
\text { (ii) Single cell resolution } \\
\text { (iii) Easy to assay axon trafficking and } \\
\text { synaptic bouton morphology (Mudher et } \\
\text { al. 2004, Talmat-Amar et al. 2011) } \\
\text { (iv) Can perform electrophysiology to } \\
\text { directly measure neuronal function (Chee } \\
\text { et al. 2005) }\end{array}$ & $\begin{array}{l}\text { (i) Cannot examine age-dependent tau- } \\
\text { mediated phenotypes in larva }\end{array}$ \\
\hline b. Sensory neurons & $\begin{array}{l}\text { c161, e16E, } \\
\text { R7 }\end{array}$ & $\begin{array}{l}\text { (i) Can be used to examine toxicity in } \\
\text { adult sensory neurons (Williams et al. } \\
\text { 2000, Sealey et al. 2017) } \\
\text { (ii) Can be used to examine age- } \\
\text { dependent tau toxicity in the adult fly eye } \\
\text { (e.g. R7) }\end{array}$ & $\begin{array}{l}\text { (i) Expression not specific to one particular type } \\
\text { of sensory neuron }\end{array}$ \\
\hline $\begin{array}{l}\text { c. Neurotransmitter } \\
\text { specific }\end{array}$ & $\begin{array}{l}\text { Cha, Ddc, } \\
\text { 996TPH, } \\
\text { TH, DvGlut, } \\
\text { Gad, NPF }\end{array}$ & $\begin{array}{l}\text { (i) Targeting expression to smaller } \\
\text { groups of neurons with a common } \\
\text { physiological property (Wu et al. 2013) } \\
\text { (ii) See tangle pathology in dopaminergic } \\
\text { neurons }\end{array}$ & $\begin{array}{l}\text { (i) Some driver lines produce more specific, } \\
\text { spatially restricted expression patterns than } \\
\text { others }\end{array}$ \\
\hline
\end{tabular}




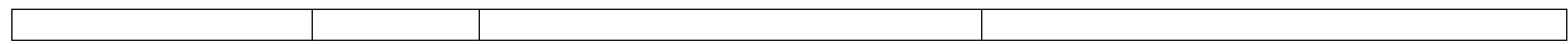

* Note models are organised by tissue-level specificity of study not GAL4 driver-level specificity of expression 


\section{References}

Ali, Y. O., K. Ruan \& R. G. Zhai (2012) NMNAT suppresses tau-induced neurodegeneration by promoting clearance of hyperphosphorylated tau oligomers in a Drosophila model of tauopathy. Hum Mol Genet, 21, 237-50.

Ambegaokar, S. S. \& G. R. Jackson (2011) Functional genomic screen and network analysis reveal novel modifiers of tauopathy dissociated from tau phosphorylation. Hum Mol Genet, 20, 4947-77.

Arendt, D., A. S. Denes, G. Jékely \& K. Tessmar-Raible (2008) The evolution of nervous system centralization. Philos Trans $R$ Soc Lond B Biol Sci, 363, 1523-8.

Arendt, D., M. A. Tosches \& H. Marlow (2016) From nerve net to nerve ring, nerve cord and brain--evolution of the nervous system. Nat Rev Neurosci, 17, 61-72.

Bakhoum, M. F., C. Y. Bakhoum, Z. Ding, S. M. Carlton, G. A. Campbell \& G. R. Jackson (2014) Evidence for autophagic gridlock in aging and neurodegeneration. Trans/ Res, 164, 1-12.

Berger, C., S. Renner, K. Lüer \& G. M. Technau (2007) The commonly used marker ELAV is transiently expressed in neuroblasts and glial cells in the Drosophila embryonic CNS. Dev Dyn, 236, 3562-8.

Bier, E. (2005) Drosophila, the golden bug, emerges as a tool for human genetics. Nat Rev Genet, 6, 9-23.

Bischof, J., R. K. Maeda, M. Hediger, F. Karch \& K. Basler (2007) An optimized transgenesis system for Drosophila using germ-line-specific phiC31 integrases. Proc Natl Acad Sci U S A, 104, 3312-7.

Brand, A. H. \& N. Perrimon (1993) Targeted gene expression as a means of altering cell fates and generating dominant phenotypes. Development, 118, 401-15.

Burnouf, S., S. Grönke, H. Augustin, J. Dols, M. K. Gorsky, J. Werner, F. Kerr, N. Alic, P. Martinez \& L. Partridge (2016) Deletion of endogenous Tau proteins is not detrimental in Drosophila. Sci Rep, 6, 23102.

Butterfield, D. A. \& J. B. Owen (2011) Lectin-affinity chromatography brain glycoproteomics and Alzheimer disease: insights into protein alterations consistent with the pathology and progression of this dementing disorder. Proteomics Clin Appl, 5, 50-6.

Butzlaff, M., S. B. Hannan, P. Karsten, S. Lenz, J. Ng, H. Voßfeldt, K. Prüßing, R. Pflanz, J. B. Schulz, T. Rasse \& A. Voigt (2015) Impaired retrograde transport by the Dynein/Dynactin complex contributes to Tau-induced toxicity. Hum Mol Genet, 24, 3623-37.

Carmine-Simmen, K., T. Proctor, J. Tschäpe, B. Poeck, T. Triphan, R. Strauss \& D. Kretzschmar (2009) Neurotoxic effects induced by the Drosophila amyloid-beta peptide suggest a conserved toxic function. Neurobiol Dis, 33, 274-81.

Castillo-Carranza, D. L., U. Sengupta, M. J. Guerrero-Muñoz, C. A. Lasagna-Reeves, J. E. Gerson, G. Singh, D. M. Estes, A. D. Barrett, K. T. Dineley, G. R. Jackson \& R. Kayed (2014) Passive immunization with Tau oligomer monoclonal antibody reverses tauopathy phenotypes without affecting hyperphosphorylated neurofibrillary tangles. J Neurosci, 34, 4260-72.

Chatterjee, S., T. K. Sang, G. M. Lawless \& G. R. Jackson (2009) Dissociation of tau toxicity and phosphorylation: role of GSK-3beta, MARK and Cdk5 in a Drosophila model. Hum Mol Genet, 18, 164-77.

Chee, F. C., A. Mudher, M. F. Cuttle, T. A. Newman, D. MacKay, S. Lovestone \& D. Shepherd (2005) Over-expression of tau results in defective synaptic transmission in Drosophila neuromuscular junctions. Neurobiol Dis, 20, 918-28. 
Chouhan, A. K., C. Guo, Y. C. Hsieh, H. Ye, M. Senturk, Z. Zuo, Y. Li, S. Chatterjee, J. Botas, G. R. Jackson, H. J. Bellen \& J. M. Shulman (2016) Uncoupling neuronal death and dysfunction in Drosophila models of neurodegenerative disease. Acta Neuropathol Commun, 4, 62.

Colodner, K. J. \& M. B. Feany (2010) Glial fibrillary tangles and JAK/STAT-mediated glial and neuronal cell death in a Drosophila model of glial tauopathy. J Neurosci, 30, 16102-13.

Cowan, C. M., T. Bossing, A. Page, D. Shepherd \& A. Mudher (2010a) Soluble hyperphosphorylated tau causes microtubule breakdown and functionally compromises normal tau in vivo. Acta Neuropathol, 120, 593-604.

Cowan, C. M., F. Chee, D. Shepherd \& A. Mudher (2010b) Disruption of neuronal function by soluble hyperphosphorylated tau in a Drosophila model of tauopathy. Biochem Soc Trans, 38, 564-70.

Cowan, C. M. \& A. Mudher (2013) Are tau aggregates toxic or protective in tauopathies? Front Neurol, 4, 114.

Cowan, C. M., S. Quraishe, S. Hands, M. Sealey, S. Mahajan, D. W. Allan \& A. Mudher (2015) Rescue from tau-induced neuronal dysfunction produces insoluble tau oligomers. Sci Rep, 5, 17191.

Denes, A. S., G. Jékely, P. R. Steinmetz, F. Raible, H. Snyman, B. Prud'homme, D. E. Ferrier, G. Balavoine \& D. Arendt (2007) Molecular architecture of annelid nerve cord supports common origin of nervous system centralization in bilateria. Cell, 129, 27788.

Dietzl, G., D. Chen, F. Schnorrer, K. C. Su, Y. Barinova, M. Fellner, B. Gasser, K. Kinsey, S. Oppel, S. Scheiblauer, A. Couto, V. Marra, K. Keleman \& B. J. Dickson (2007) A genome-wide transgenic RNAi library for conditional gene inactivation in Drosophila. Nature, 448, 151-6.

Dolan, P. J. \& G. V. Johnson (2010) The role of tau kinases in Alzheimer's disease. Curr Opin Drug Discov Devel, 13, 595-603.

Dourlen, P., F. J. Fernandez-Gomez, C. Dupont, B. Grenier-Boley, C. Bellenguez, H. Obriot, R. Caillierez, Y. Sottejeau, J. Chapuis, A. Bretteville, F. Abdelfettah, C. Delay, N. Malmanche, H. Soininen, M. Hiltunen, M. C. Galas, P. Amouyel, N. Sergeant, L. Buée, J. C. Lambert \& B. Dermaut (2017) Functional screening of Alzheimer risk loci identifies PTK2B as an in vivo modulator and early marker of Tau pathology. Mol Psychiatry, 22, 874-883.

DuBoff, B., J. Götz \& M. B. Feany (2012) Tau promotes neurodegeneration via DRP1 mislocalization in vivo. Neuron, 75, 618-32.

Feany, M. B. \& W. W. Bender (2000) A Drosophila model of Parkinson's disease. Nature, 404, 394-8.

Fernius, J., A. Starkenberg, M. Pokrzywa \& S. Thor (2017) Human TTBK1, TTBK2 and MARK1 kinase toxicity in. Biol Open, 6, 1013-1023.

Folwell, J., C. M. Cowan, K. K. Ubhi, H. Shiabh, T. A. Newman, D. Shepherd \& A. Mudher (2010) Abeta exacerbates the neuronal dysfunction caused by human tau expression in a Drosophila model of Alzheimer's disease. Exp Neurol, 223, 401-9.

Fouquet, W., D. Owald, C. Wichmann, S. Mertel, H. Depner, M. Dyba, S. Hallermann, R. J. Kittel, S. Eimer \& S. J. Sigrist (2009) Maturation of active zone assembly by Drosophila Bruchpilot. J Cell Biol, 186, 129-45.

Freeman, M. (1996) Reiterative use of the EGF receptor triggers differentiation of all cell types in the Drosophila eye. Cell, 87, 651-60.

Frenkel-Pinter, M., S. Stempler, S. Tal-Mazaki, Y. Losev, A. Singh-Anand, D. EscobarÁlvarez, J. Lezmy, E. Gazit, E. Ruppin \& D. Segal (2017) Altered protein 
glycosylation predicts Alzheimer's disease and modulates its pathology in disease model Drosophila. Neurobiol Aging, 56, 159-171.

Frost, B., F. H. Bardai \& M. B. Feany (2016) Lamin Dysfunction Mediates Neurodegeneration in Tauopathies. Curr Biol, 26, 129-36.

Fulga, T. A., I. Elson-Schwab, V. Khurana, M. L. Steinhilb, T. L. Spires, B. T. Hyman \& M. B. Feany (2007) Abnormal bundling and accumulation of F-actin mediates tauinduced neuronal degeneration in vivo. Nat Cell Biol, 9, 139-48.

Gorsky, M. K., S. Burnouf, O. Sofola-Adesakin, J. Dols, H. Augustin, C. M. Weigelt, S. Grönke \& L. Partridge (2017) Pseudo-acetylation of multiple sites on human Tau proteins alters Tau phosphorylation and microtubule binding, and ameliorates amyloid beta toxicity. Sci Rep, 7, 9984.

Grammenoudi, S., S. Kosmidis \& E. M. Skoulakis (2006) Cell type-specific processing of human Tau proteins in Drosophila. FEBS Lett, 580, 4602-6.

Grundke-lqbal, I., K. Iqbal, Y. C. Tung, M. Quinlan, H. M. Wisniewski \& L. I. Binder (1986) Abnormal phosphorylation of the microtubule-associated protein tau (tau) in Alzheimer cytoskeletal pathology. Proc Natl Acad Sci U S A, 83, 4913-7.

Harris, R. M., B. D. Pfeiffer, G. M. Rubin \& J. W. Truman (2015) Neuron hemilineages provide the functional ground plan for the Drosophila ventral nervous system. Elife, 4.

Heidary, G. \& M. E. Fortini (2001) Identification and characterization of the Drosophila tau homolog. Mech Dev, 108, 171-8.

Iqbal, K., A. e. C. Alonso \& I. Grundke-lqbal (2008) Cytosolic abnormally hyperphosphorylated tau but not paired helical filaments sequester normal MAPs and inhibit microtubule assembly. J Alzheimers Dis, 14, 365-70.

Ito, K., H. Sass, J. Urban, A. Hofbauer \& S. Schneuwly (1997) GAL4-responsive UAS-tau as a tool for studying the anatomy and development of the Drosophila central nervous system. Cell Tissue Res, 290, 1-10.

Jackson, G. R., I. Salecker, X. Dong, X. Yao, N. Arnheim, P. W. Faber, M. E. MacDonald \& S. L. Zipursky (1998) Polyglutamine-expanded human huntingtin transgenes induce degeneration of Drosophila photoreceptor neurons. Neuron, 21, 633-42.

Jackson, G. R., M. Wiedau-Pazos, T. K. Sang, N. Wagle, C. A. Brown, S. Massachi \& D. H. Geschwind (2002) Human wild-type tau interacts with wingless pathway components and produces neurofibrillary pathology in Drosophila. Neuron, 34, 509-19.

Jenett, A., G. M. Rubin, T. T. Ngo, D. Shepherd, C. Murphy, H. Dionne, B. D. Pfeiffer, A. Cavallaro, D. Hall, J. Jeter, N. Iyer, D. Fetter, J. H. Hausenfluck, H. Peng, E. T. Trautman, R. R. Svirskas, E. W. Myers, Z. R. Iwinski, Y. Aso, G. M. DePasquale, A. Enos, P. Hulamm, S. C. Lam, H. H. Li, T. R. Laverty, F. Long, L. Qu, S. D. Murphy, K. Rokicki, T. Safford, K. Shaw, J. H. Simpson, A. Sowell, S. Tae, Y. Yu \& C. T. Zugates (2012) A GAL4-driver line resource for Drosophila neurobiology. Cell Rep, 2, 991-1001.

Jinek, M., K. Chylinski, I. Fonfara, M. Hauer, J. A. Doudna \& E. Charpentier (2012) A programmable dual-RNA-guided DNA endonuclease in adaptive bacterial immunity. Science, 337, 816-21.

Jordán-Álvarez, S., W. Fouquet, S. J. Sigrist \& A. Acebes (2012) Presynaptic PI3K activity triggers the formation of glutamate receptors at neuromuscular terminals of Drosophila. J Cell Sci, 125, 3621-9.

Kanninen, K., G. Goldsteins, S. Auriola, I. Alafuzoff \& J. Koistinaho (2004) Glycosylation changes in Alzheimer's disease as revealed by a proteomic approach. Neurosci Lett, 367, 235-40. 
Kardon, J. R. \& R. D. Vale (2009) Regulators of the cytoplasmic dynein motor. Nat Rev Mol Cell Biol, 10, 854-65.

Karsten, S. L., T. K. Sang, L. T. Gehman, S. Chatterjee, J. Liu, G. M. Lawless, S. Sengupta, R. W. Berry, J. Pomakian, H. S. Oh, C. Schulz, K. S. Hui, M. Wiedau-Pazos, H. V. Vinters, L. I. Binder, D. H. Geschwind \& G. R. Jackson (2006) A genomic screen for modifiers of tauopathy identifies puromycin-sensitive aminopeptidase as an inhibitor of tau-induced neurodegeneration. Neuron, 51, 549-60.

Keshishian, H., K. Broadie, A. Chiba \& M. Bate (1996) The drosophila neuromuscular junction: a model system for studying synaptic development and function. Annu Rev Neurosci, 19, 545-75.

Khurana, V., Y. Lu, M. L. Steinhilb, S. Oldham, J. M. Shulman \& M. B. Feany (2006) TORmediated cell-cycle activation causes neurodegeneration in a Drosophila tauopathy model. Curr Biol, 16, 230-41.

Kimura, T., S. Yamashita, T. Fukuda, J. M. Park, M. Murayama, T. Mizoroki, Y. Yoshiike, N. Sahara \& A. Takashima (2007) Hyperphosphorylated tau in parahippocampal cortex impairs place learning in aged mice expressing wild-type human tau. EMBO J, 26, 5143-52.

Koh, Y. H., E. Popova, U. Thomas, L. C. Griffith \& V. Budnik (1999) Regulation of DLG localization at synapses by CaMKII-dependent phosphorylation. Cell, 98, 353-63.

Kosmidis, S., S. Grammenoudi, K. Papanikolopoulou \& E. M. Skoulakis (2010) Differential effects of Tau on the integrity and function of neurons essential for learning in Drosophila. J Neurosci, 30, 464-77.

Ksiezak-Reding, H., L. I. Binder \& S. H. Yen (1988) Immunochemical and biochemical characterization of tau proteins in normal and Alzheimer's disease brains with Alz 50 and Tau-1. J Biol Chem, 263, 7948-53.

Lacin, H. \& J. W. Truman (2016) Lineage mapping identifies molecular and architectural similarities between the larval and adult Drosophila central nervous system. Elife, 5, e13399.

Lasagna-Reeves, C. A., M. de Haro, S. Hao, J. Park, M. W. Rousseaux, I. Al-Ramahi, P. Jafar-Nejad, L. Vilanova-Velez, L. See, A. De Maio, L. Nitschke, Z. Wu, J. C. Troncoso, T. F. Westbrook, J. Tang, J. Botas \& H. Y. Zoghbi (2016) Reduction of Nuak1 Decreases Tau and Reverses Phenotypes in a Tauopathy Mouse Model. Neuron, 92, 407-418.

Lee, T., A. Lee \& L. Luo (1999) Development of the Drosophila mushroom bodies: sequential generation of three distinct types of neurons from a neuroblast. Development, 126, 4065-76.

Luan, H., N. C. Peabody, C. R. Vinson \& B. H. White (2006) Refined spatial manipulation of neuronal function by combinatorial restriction of transgene expression. Neuron, 52, 425-36.

Lupo, G., W. A. Harris \& K. E. Lewis (2006) Mechanisms of ventral patterning in the vertebrate nervous system. Nat Rev Neurosci, 7, 103-14.

Malmanche, N., P. Dourlen, M. Gistelinck, F. Demiautte, N. Link, C. Dupont, L. Vanden Broeck, E. Werkmeister, P. Amouyel, A. Bongiovanni, H. Bauderlique, D. Moechars, A. Royou, H. J. Bellen, F. Lafont, P. Callaerts, J. C. Lambert \& B. Dermaut (2017) Developmental Expression of 4-Repeat-Tau Induces Neuronal Aneuploidy in Drosophila Tauopathy Models. Sci Rep, 7, 40764.

Mavilia, C., D. Couchie, M. G. Mattei, M. P. Nivez \& J. Nunez (1993) High and low molecular weight tau proteins are differentially expressed from a single gene. $J$ Neurochem, 61, 1073-81. 
McGuire, S. E., Z. Mao \& R. L. Davis (2004) Spatiotemporal gene expression targeting with the TARGET and gene-switch systems in Drosophila. Sci STKE, 2004, pl6.

McMillan, P., E. Korvatska, P. Poorkaj, Z. Evstafjeva, L. Robinson, L. Greenup, J. Leverenz, G. D. Schellenberg \& I. D'Souza (2008) Tau isoform regulation is regionand cell-specific in mouse brain. J Comp Neurol, 511, 788-803.

Menon, K. P., R. A. Carrillo \& K. Zinn (2013) Development and plasticity of the Drosophila larval neuromuscular junction. Wiley Interdiscip Rev Dev Biol, 2, 647-70.

Mershin, A., E. Pavlopoulos, O. Fitch, B. C. Braden, D. V. Nanopoulos \& E. M. Skoulakis (2004) Learning and memory deficits upon TAU accumulation in Drosophila mushroom body neurons. Learn Mem, 11, 277-87.

Mudher, A., D. Shepherd, T. A. Newman, P. Mildren, J. P. Jukes, A. Squire, A. Mears, J. A. Drummond, S. Berg, D. MacKay, A. A. Asuni, R. Bhat \& S. Lovestone (2004) GSK3beta inhibition reverses axonal transport defects and behavioural phenotypes in Drosophila. Mol Psychiatry, 9, 522-30.

Ni, J. Q., M. Markstein, R. Binari, B. Pfeiffer, L. P. Liu, C. Villalta, M. Booker, L. Perkins \& N. Perrimon (2008) Vector and parameters for targeted transgenic RNA interference in Drosophila melanogaster. Nat Methods, 5, 49-51.

Nicolaï, L. J., A. Ramaekers, T. Raemaekers, A. Drozdzecki, A. S. Mauss, J. Yan, M. Landgraf, W. Annaert \& B. A. Hassan (2010) Genetically encoded dendritic marker sheds light on neuronal connectivity in Drosophila. Proc Natl Acad Sci U S A, 107, 20553-8.

Nishimura, I., Y. Yang \& B. Lu (2004) PAR-1 kinase plays an initiator role in a temporally ordered phosphorylation process that confers tau toxicity in Drosophila. Cell, 116, 671-82.

Osterwalder, T., K. S. Yoon, B. H. White \& H. Keshishian (2001) A conditional tissuespecific transgene expression system using inducible GAL4. Proc Natl Acad Sci U S A, 98, 12596-601.

Papanikolopoulou, K., S. Kosmidis, S. Grammenoudi \& E. M. Skoulakis (2010) Phosphorylation differentiates tau-dependent neuronal toxicity and dysfunction. Biochem Soc Trans, 38, 981-7.

Papanikolopoulou, K. \& E. M. Skoulakis (2015) Temporally distinct phosphorylations differentiate Tau-dependent learning deficits and premature mortality in Drosophila. Hum Mol Genet, 24, 2065-77.

Passarella, D. \& M. Goedert (2018) Beta-sheet assembly of Tau and neurodegeneration in Drosophila melanogaster. Neurobiol Aging, 72, 98-105.

Pfeiffer, B. D., A. Jenett, A. S. Hammonds, T. T. Ngo, S. Misra, C. Murphy, A. Scully, J. W. Carlson, K. H. Wan, T. R. Laverty, C. Mungall, R. Svirskas, J. T. Kadonaga, C. Q. Doe, M. B. Eisen, S. E. Celniker \& G. M. Rubin (2008) Tools for neuroanatomy and neurogenetics in Drosophila. Proc Natl Acad Sci U S A, 105, 9715-20.

Pfeiffer, B. D., T. T. Ngo, K. L. Hibbard, C. Murphy, A. Jenett, J. W. Truman \& G. M. Rubin (2010) Refinement of tools for targeted gene expression in Drosophila. Genetics, 186, 735-55.

Phelps, C. B. \& A. H. Brand (1998) Ectopic gene expression in Drosophila using GAL4 system. Methods, 14, 367-79.

Philippidou, P. \& J. S. Dasen (2013) Hox genes: choreographers in neural development, architects of circuit organization. Neuron, 80, 12-34.

Pilling, A. D., D. Horiuchi, C. M. Lively \& W. M. Saxton (2006) Kinesin-1 and Dynein are the primary motors for fast transport of mitochondria in Drosophila motor axons. Mol Biol Cell, 17, 2057-68. 
Quraishe, S., C. M. Cowan \& A. Mudher (2013) NAP (davunetide) rescues neuronal dysfunction in a Drosophila model of tauopathy. Mol Psychiatry, 18, 834-42.

Quraishe, S., M. Sealey, L. Cranfield \& A. Mudher (2016) Microtubule stabilising peptides rescue tau phenotypes in-vivo. Sci Rep, 6, 38224.

--- (2017) Corrigendum: Microtubule stabilising peptides rescue tau phenotypes in-vivo. Sci Rep, 7, 40833.

Riedl, J., A. H. Crevenna, K. Kessenbrock, J. H. Yu, D. Neukirchen, M. Bista, F. Bradke, D. Jenne, T. A. Holak, Z. Werb, M. Sixt \& R. Wedlich-Soldner (2008) Lifeact: a versatile marker to visualize F-actin. Nat Methods, 5, 605-7.

Robinson, I. M., R. Ranjan \& T. L. Schwarz (2002) Synaptotagmins I and IV promote transmitter release independently of $\mathrm{Ca}(2+)$ binding in the $\mathrm{C}(2) \mathrm{A}$ domain. Nature, 418, 336-40.

Rolls, M. M., D. Satoh, P. J. Clyne, A. L. Henner, T. Uemura \& C. Q. Doe (2007) Polarity and intracellular compartmentalization of Drosophila neurons. Neural Dev, 2, 7.

Roy, B. \& G. R. Jackson (2014) Interactions between Tau and a-synuclein augment neurotoxicity in a Drosophila model of Parkinson's disease. Hum Mol Genet, 23, 3008-23.

Schedin-Weiss, S., B. Winblad \& L. O. Tjernberg (2014) The role of protein glycosylation in Alzheimer disease. FEBS J, 281, 46-62.

Sealey, M. A., E. Vourkou, C. M. Cowan, T. Bossing, S. Quraishe, S. Grammenoudi, E. M. C. Skoulakis \& A. Mudher (2017) Distinct phenotypes of three-repeat and four-repeat human tau in a transgenic model of tauopathy. Neurobiol Dis, 105, 74-83.

Shulman, J. M., P. Chipendo, L. B. Chibnik, C. Aubin, D. Tran, B. T. Keenan, P. L. Kramer, J. A. Schneider, D. A. Bennett, M. B. Feany \& P. L. De Jager (2011) Functional screening of Alzheimer pathology genome-wide association signals in Drosophila. Am J Hum Genet, 88, 232-8.

Sims, N. R., D. M. Bowen, S. J. Allen, C. C. Smith, D. Neary, D. J. Thomas \& A. N. Davison (1983) Presynaptic cholinergic dysfunction in patients with dementia. J Neurochem, 40, 503-9.

Sivanantharajah, L. \& B. Zhang (2015) Current techniques for high-resolution mapping of behavioral circuits in Drosophila. J Comp Physiol A Neuroethol Sens Neural Behav Physiol, 201, 895-909.

Sotiropoulos, I., M. C. Galas, J. M. Silva, E. Skoulakis, S. Wegmann, M. B. Maina, D. Blum, C. L. Sayas, E. M. Mandelkow, E. Mandelkow, M. G. Spillantini, N. Sousa, J. Avila, M. Medina, A. Mudher \& L. Buee (2017) Atypical, non-standard functions of the microtubule associated Tau protein. Acta Neuropathol Commun, 5, 91.

Sweeney, N. T., J. E. Brenman, Y. N. Jan \& F. B. Gao (2006) The coiled-coil protein shrub controls neuronal morphogenesis in Drosophila. Curr Biol, 16, 1006-11.

Takemura, S. Y., Y. Aso, T. Hige, A. Wong, Z. Lu, C. S. Xu, P. K. Rivlin, H. Hess, T. Zhao, T. Parag, S. Berg, G. Huang, W. Katz, D. J. Olbris, S. Plaza, L. Umayam, R. Aniceto, L. A. Chang, S. Lauchie, O. Ogundeyi, C. Ordish, A. Shinomiya, C. Sigmund, S. Takemura, J. Tran, G. C. Turner, G. M. Rubin \& L. K. Scheffer (2017a) A connectome of a learning and memory center in the adult. Elife, 6.

Takemura, S. Y., A. Nern, D. B. Chklovskii, L. K. Scheffer, G. M. Rubin \& I. A. Meinertzhagen (2017b) The comprehensive connectome of a neural substrate for 'ON' motion detection in. Elife, 6.

Talmat-Amar, Y., Y. Arribat, C. Redt-Clouet, S. Feuillette, A. L. Bougé, M. Lecourtois \& M. L. Parmentier (2011) Important neuronal toxicity of microtubule-bound Tau in vivo in Drosophila. Hum Mol Genet, 20, 3738-45. 
Tomlinson, A., D. D. Bowtell, E. Hafen \& G. M. Rubin (1987) Localization of the sevenless protein, a putative receptor for positional information, in the eye imaginal disc of Drosophila. Cell, 51, 143-50.

Ugur, B., K. Chen \& H. J. Bellen (2016) Drosophila tools and assays for the study of human diseases. Dis Model Mech, 9, 235-44.

Vagnoni, A. \& S. L. Bullock (2016) A simple method for imaging axonal transport in aging neurons using the adult Drosophila wing. Nat Protoc, 11, 1711-23.

Williams, D. W., M. Tyrer \& D. Shepherd (2000) Tau and tau reporters disrupt central projections of sensory neurons in Drosophila. J Comp Neurol, 428, 630-40.

Wittmann, C. W., M. F. Wszolek, J. M. Shulman, P. M. Salvaterra, J. Lewis, M. Hutton \& M. B. Feany (2001) Tauopathy in Drosophila: neurodegeneration without neurofibrillary tangles. Science, 293, 711-4.

Wolff, G. H. \& N. J. Strausfeld (2016) Genealogical correspondence of a forebrain centre implies an executive brain in the protostome-deuterostome bilaterian ancestor. Philos Trans $R$ Soc Lond B Biol Sci, 371, 20150055.

Wu, T. H., Y. N. Lu, C. L. Chuang, C. L. Wu, A. S. Chiang, D. E. Krantz \& H. Y. Chang (2013) Loss of vesicular dopamine release precedes tauopathy in degenerative dopaminergic neurons in a Drosophila model expressing human tau. Acta Neuropathol, 125, 711-25.

Zheng, Y., J. Wildonger, B. Ye, Y. Zhang, A. Kita, S. H. Younger, S. Zimmerman, L. Y. Jan \& Y. N. Jan (2008) Dynein is required for polarized dendritic transport and uniform microtubule orientation in axons. Nat Cell Biol, 10, 1172-80.

Zhu, Y., X. Shan, S. A. Yuzwa \& D. J. Vocadlo (2014) The emerging link between OGlcNAc and Alzheimer disease. J Biol Chem, 289, 34472-81.

Šimić, G., M. Babić Leko, S. Wray, C. Harrington, I. Delalle, N. Jovanov-Milošević, D. Bažadona, L. Buée, R. de Silva, G. Di Giovanni, C. Wischik \& P. R. Hof (2016) Tau Protein Hyperphosphorylation and Aggregation in Alzheimer's Disease and Other Tauopathies, and Possible Neuroprotective Strategies. Biomolecules, 6, 6. 